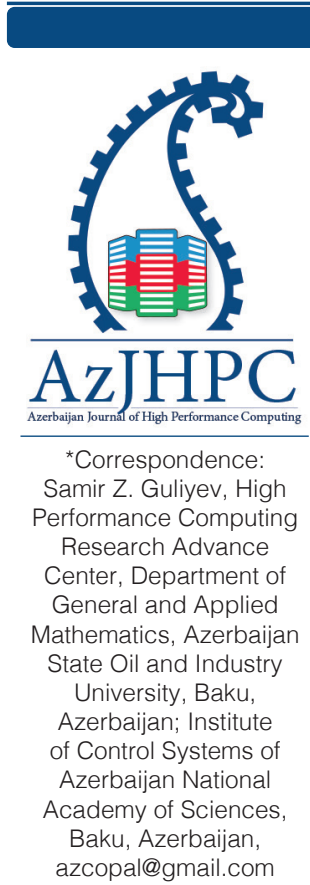

\title{
Synthesis of Zonal Control of Lumped Sources for the Heat Conduction Process
}

Samir Z. Guliyev

High Performance Computing Research Advance Center, Department of General and Applied Mathematics, Azerbaijan State Oil and Industry University, Baku, Azerbaijan; Institute of Control Systems of Azerbaijan National Academy of Sciences, Baku, Azerbaijan, azcopal@gmail.com

\section{Abstract}

The paper studies the problem of synthesis of control of lumped sources for an object with distributed parameters based on discrete observation of the phase state at specific object points. We propose an approach in which the whole phase space at the observed points is preliminarily divided in some way into given subsets (zones). The synthesized controls are selected from the class of piecewise-constant functions, and their current values are determined by a subset of the phase space containing the population of current states of the object at the observed points, at which controls take constant values. Such synthesized controls are called zonal. We give a numerical technique for obtaining optimal values of zonal controls using efficient first-order optimization methods. To this purpose, we derive formulas for the gradient of the objective function in the space of zonal controls.

Keyword: Synthesis of Control, Zonal Control, Feedback, Distributed System, Heat Equation, the Gradient of Functional.

\section{Introduction}

It is known that one of the important areas in the modern theory of automatic control is the theory of control of systems with distributed parameters. The problems of synthesis of distributed control systems are, in most cases, more complex than lumped systems due to the characteristics of distributed objects. Distributed control objects include many chemical-technological, radiation, aerodynamic and hydrodynamic processes, heat conduction and diffusion processes, processes associated with the movement of elastic structures, etc. The absence of a formalized methodological approach for solving problems of controlling objects with distributed parameters poses certain problems for researchers that require the use of non-standard research methods and decision-making in each specific case. The main contribution to the development of the theory of distributed-parameter control systems has been provided by a number of fundamental results obtained in the works of A.G. Butkovsky (1975), A.I. Egorov (2004), T.K. Sirazetdinov (1977), J.L. Lyons (1971), N.N. Moiseev (1971), K.A. Lurie (1993), E.Ya. Rapoport (1999), (see also Fursikov A.V., 1999; Arthur E. Bryson, Yu-Chi Ho., 1975), etc. Modern technical means of measuring and computing technology, which make it possible to carry out a large volume of measuring and computational work in real-time, have played a key role in the development of feedback 
control systems and their widespread practical implementation.

The paper considers the problem of synthesizing control of an object with distributed parameters on special classes of control actions. For the synthesized controls, the concept of zoning is introduced, which means the constancy of the values of the synthesized control parameters in each of the subsets (zones), into which the entire set of possible states of the object or the time interval of the functioning of the process (object) is pre-divided. The control actions' values are also determined by the type of feedback and the class of the functional dependence of the control on the currently observed value of the state or the current time of observation. Particularly, the case of discrete feedback is analyzed using discrete observation of the phase state of the object at its certain points.

The constancy of the parameters of the zonal control actions (the zonal controls themselves or the zonal amplification coefficients under a linear dependence of the control on the state of the process) determines the robustness of the control system, as well as ensures the feasibility of synthesized control actions with sufficiently high accuracy and improves the technical performance of the equipment involved in the control loop.

We have used the principle of zoning of control parameters as the basis of numerical methods for solving such specific optimization and inverse problems like the problem of optimal placement of production and injection wells and optimal control of their flow rates during the operation of an oil reservoir under the regime of water-driven piston displacement (Guliyev S.Z., Aida-zade K.R., 2005), and the problem of identifying the hydraulic resistance coefficient under the unsteady flow of viscous fluids through pipelines (Guliyev S.Z. and Aida-zade K.R., 2016), as well as problems of synthesis of control and identification of objects with lumped parameters (Kuliev, S. Z., 2011; Aida-Zade, K. R., \& Kuliev, S. Z., 2012; Aida-Zade, K. R., \& Kuliev, S. Z., 2011; Guliyev, S. Z. (2013).

\section{Problem Statement}

To illustrate the proposed approach, we consider the problem of controlling a rod heating process through lumped point sources. This process can be described by the following parabolic type partial differential equation:

$$
u_{t}=a^{2} u_{x x}+\sum_{j=1}^{M} \vartheta^{j}(t) \delta\left(x-\bar{x}^{j}\right), \quad(x, t) \in \Omega=(0,1) \times(0, T]
$$

Here $[0,1]$ is the segment occupied by the rod; $\bar{x}^{j}(j=1,2, \ldots, M)$ the points at which heat sources with optimizable powers $\vartheta^{j}(t)(j=1,2, \ldots, M)$ are placed; $M$ the given number of heat sources; $\delta\left(\right.$. ) the one-dimensional generalized Dirac's function; $a^{2}$ the thermal diffusivity. Initial and boundary conditions are given in the following form:

$$
\begin{aligned}
& u(x, 0)=g_{0}(x) \in G_{0}(x), x \in[0,1], \\
& u(0, t)=g_{1}(t) \in G_{1}(t), t \in[0, T], \\
& u(1, t)=g_{2}(t) \in G_{2}(t), t \in[0, T],
\end{aligned}
$$


Here $G_{0}(),. G_{1}($.$) , and G_{2}($.$) are point-set mappings, for which each value of the$ argument is associated with a closed bounded set. In this case, the corresponding distribution functions $\Phi_{0}\left(g_{0}\right), \Phi_{1}\left(g_{1}\right)$, and $\Phi_{2}\left(g_{2}\right)$ are given, which characterize the distributions of possible values that the initial and boundary conditions can take.

Assume that thermal sensors are installed at $N$ points of the rod with coordinates $\tilde{x}^{s}(s=1,2, \ldots, N)$. These sensors realize operative observation and input to the control system of information on the state of the heating process at these points, which is determined by the vector:

$$
\tilde{u}(t)=\left(\tilde{u}^{1}(t), \tilde{u}^{2}(t), \ldots, \tilde{u}^{N}(t)\right)^{*}=\left(u\left(\tilde{x}^{1}, t\right), u\left(\tilde{x}^{2}, t\right), \ldots, u\left(\tilde{x}^{N}, t\right)\right)^{*}, \quad t \in(0, T],
$$

where ${ }^{*}$ denotes the transposition sign. Besides, the discrete points of observation time $\tau_{i} \in[0, T](i=0,1, \ldots, q)$ are given, at which it is possible to measure the value of the object's state at the points of the rod where the thermal sensors are installed, i.e. $\tilde{u}(t)$.

To control the heat conduction process in the rod, it is required to synthesize a regulator that, based on the results of temperature measurements at the points $\tilde{x}^{s}$ ( $s=$ $1,2, \ldots, N)$ of the rod, would ensure the maintenance of the temperature $u(x, T)$ at a given level by maintaining the required temperature $\vartheta(t)$ in the heat sources. Based on technological conditions, we have to impose certain constraints on the values that the controls can take:

$$
V^{j}=\left\{\vartheta(t): \vartheta_{\min }^{j} \leq \vartheta^{j}(t) \leq \vartheta_{\max }\right\}, \quad j=1,2, \ldots, M,
$$

where $\vartheta_{\min }^{j}$ and $\vartheta_{\max }$ are given values. Here $V^{j}$ is the set of permissible values of the control $\vartheta^{j}(t)$ and $V=\left(V^{1}, V^{2}, \ldots, V^{M}\right)^{*}$.

The considered feedback control problem for the rod heating process consists in choosing permissible values of the sources' powers as a function of the object's state values

$$
\vartheta^{j}(t)=\vartheta^{j}(\tilde{u}(t)), \quad \vartheta^{j}(t) \in V^{j}, \quad j=1,2, \ldots, M, \quad t \in(0, T],
$$

at the observed points of the rod in order to minimize the given function. In the case of non-fixed initial and boundary conditions (2)-(4), the functional can take on the form:

$$
\begin{gathered}
J(\vartheta)=\iint_{G_{0}} \int_{G_{1}} I\left(x, T ; \vartheta, g_{0}, g_{1}, g_{2}\right) \mathrm{d} \Phi_{2}\left(g_{2}\right) \mathrm{d} \Phi_{1}\left(g_{1}\right) \mathrm{d} \Phi_{0}\left(g_{0}\right) \\
I\left(x, T ; \vartheta, g_{0}, g_{1}, g_{2}\right)=\int_{0}^{1}\left[u\left(x, T ; \vartheta, g_{0}, g_{1}, g_{2}\right)-\hat{u}(x)\right]^{2} \mathrm{~d} x
\end{gathered}
$$

where $u\left(x, T ; \vartheta, g_{0}, g_{1}, g_{2}\right)$ is the solution of the problem (1)-(4), corresponding to specifically chosen initial and boundary functions $g_{0}(x) \in G_{0}(x), g_{1}(t) \in G_{1}(t)$, and $g_{2}(t) \in G_{2}(t)$, and to permissible values of the controls $\vartheta(t) \in V ; \hat{u}(x)$ the given function characterizing the desired temperature distribution at the final moment of the heating process. Functional (6) characterizes the quality of process control on average 
over the sets of all possible initial states $G_{0}($.$) and boundary conditions G_{1}(),. G_{2}($.$) .$

Let the phase state values of the rod satisfy the inequalities

$$
u_{\min } \leq u\left(x, t ; \vartheta, g_{0}, g_{1}, g_{2}\right) \leq u_{\max }, \quad(x, t) \in \Omega,
$$

under all possible permissible values of the controls, as well as initial and boundary conditions. Given the points $u_{k}(k=0,1,2, \ldots, m)$, we divide the range of all possible temperature values $\left[u_{\min }, u_{\max }\right]$ into $m$ temperature intervals:

$$
\left[u_{\min }, u_{\max }\right]=\bigcup_{k=1}^{m}\left[u_{k-1}, u_{k}\right), \quad u_{0}=u_{\min }, \quad u_{m}=u_{\max } .
$$

In the $N$-dimensional phase space $\tilde{u}(t) \in R^{N}$ of the current measured temperature values at the points of the rod, we introduce the following $N$-dimensional parallelepipeds (zones):

$$
\begin{aligned}
& P_{i_{1}, i_{2}, \ldots, i_{N}}=\left\{\left(u_{1}, u_{2}, \ldots, u_{N}\right): u_{i_{s}-1} \leq u\left(\tilde{x}^{s}, t ; \vartheta, g_{0}, g_{1}, g_{2}\right) \leq u_{i_{s}}\right\}, \\
& i_{s} \in\{1,2, \ldots, m\}, s=1,2, \ldots, N,
\end{aligned}
$$

the total number of which is $m^{N}$. Let I denote the $N$-dimensional multi-index $\mathrm{I}=$ $\left(i_{1}, i_{2}, \ldots, i_{N}\right)$, which determines the number of the corresponding parallelepiped. The values of the controls $\vartheta(t)$ constant for $t \in\left[\tau_{i}, \tau_{i+1}\right)$ are determined depending on the last measured value of the observation vector over the current object's state $\tilde{u}(t)$, namely, depending on the number (multi-index) of the parallelepiped (8), to which the last measured (observed) object's state $\tilde{u}(t)$ belongs. To each phase parallelepiped there corresponds its constant control value:

$$
\begin{gathered}
\vartheta^{j}(t)=\vartheta_{i_{1}, i_{2}, \ldots, i_{N}}^{j}=\vartheta_{\mathrm{I}}^{j}=\text { const if } \tilde{u}(t) \in P_{i_{1}, i_{2}, \ldots, i_{N}}=P_{\mathrm{I}} \text { while } t \in\left[\tau_{i}, \tau_{i+1}\right) \\
j=1,2, \ldots, M .
\end{gathered}
$$

If the observed object's state belongs to the border of any zones, we use the value of the zone control of that adjacent zone into which the trajectory has passed. The number of different values that each source's power can take is equal to the number of phase parallelepipeds, i.e., $m^{N}$.

The possible configuration of phase parallelepipeds when there are only two thermal sensors is illustrated in figure 1. The phase parallelepipeds in case of three thermal sensors installed may represent cubes, etc.

It is clear that the controls (9), like (5), assume feedback. In the case of (9), the values of the controlled sources' powers during the rod heating process change only at the moments when the population of states at the observed points proceeds from one phase parallelepiped (8) to another. The total number of optimizable parameters

$$
\vartheta=\left(\vartheta_{i_{1}, i_{2}, \ldots, i_{N}}^{j}\right), \quad i_{s} \in\{1,2, \ldots, m\}, \quad s=1,2, \ldots, N, \quad j=1,2, \ldots, M,
$$

is thus equal to $p=M \times m^{N}$. 


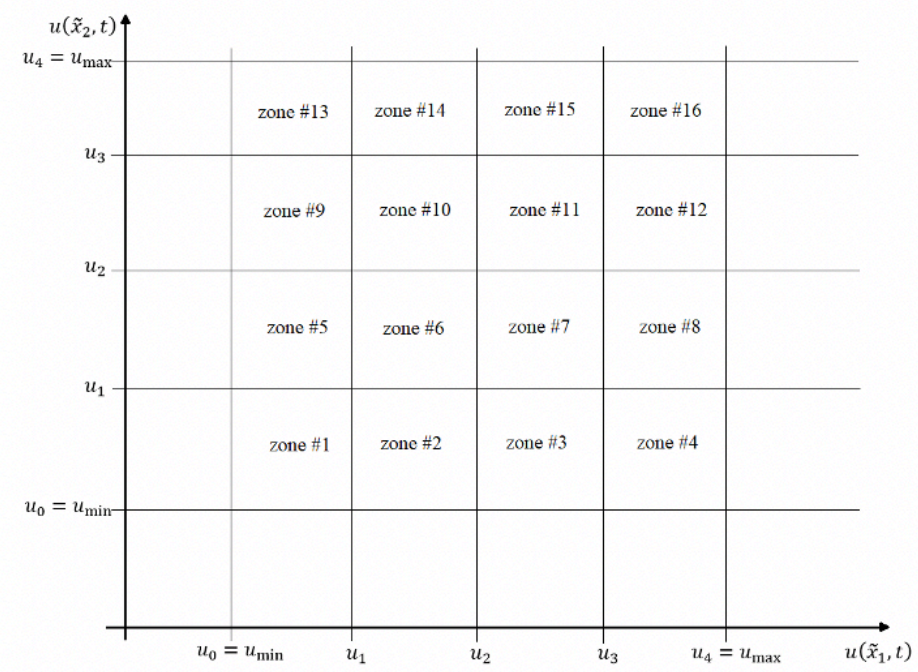

Fig. 1. Two-dimensional phase parallelepipeds in the form of rectangles

They determine the sources' behavior under all possible states of the rod at the observed points that can occur under various admissible initial and boundary conditions and control actions, on which depends the belonging of the current state $\tilde{u}(t)$ to one or another phase parallelepiped (8). Thus, the considered problem of controlling the rod heating process on the class of piecewise-constant functions with the use of feedback consists of optimizing the $M \times m^{N}$-dimensional vector $\vartheta$. The considered feedback control problem (1)-(9) is a parametric optimal control problem for a system with distributed parameters. Its specific features are, firstly, the absence of specifically prescribed initial and boundary conditions, secondly, the finitedimensionality of the sought-for control vector, and thirdly, the control is formed depending on the values of the current state of the process at the measurement points, and more precisely, depends on the multi-index defining the parallelepiped (zone) of the phase space to which the current measurement values belong. The solution of the control problem in the considered formulation (1)-(9) are synthesized zonal controls provided that the feedback with the object (process) and the choice of the values of control actions is carried out only at specified discrete moments. As examples of practical applications of such problems, one can cite the control of many technological processes and technical objects. The organization of continuous monitoring of the state is impossible, and each observation (feedback) requires specific measures and, therefore, costs time and material.

The formulated problem of synthesizing zonal controls (1)-(9) leads to a finitedimensional optimization problem. For the numerical solution of this problem, we propose to use the approach described in (Guliyev, S. Z., 2018). To solve the problem in the case of a simple design of a set of admissible controls $V$ (for example, a parallelepiped, a hyper-sphere, a polyhedron, etc.), it is effective use first-order 
parallelepiped, a hyper-sphere, a polyhedron, etc.), it is effective use first-order numerical optimization methods such as gradient projection or conjugate gradient projection methods. For example, for the conjugate gradient projection method, we construct a minimizing sequence $\left\{\vartheta^{\mathrm{k}}\right\}$ in this fashion (Vasiliev F.P., 2002; Nocedal, J., \& Wright, S., 2006):

$$
\begin{gathered}
\vartheta^{\mathrm{k}+1}=\vartheta^{\mathrm{k}}+\mathrm{P}_{(V)}\left(\vartheta^{\mathrm{k}}+\alpha_{\mathrm{k}} \mathrm{S}^{\mathrm{k}}\right), \alpha_{\mathrm{k}}>0, \mathrm{k}=0,1,2, \ldots, \mathrm{S}^{0}=-\nabla J\left(\vartheta^{0}\right), \\
\mathrm{S}^{\mathrm{k}+1}=-\nabla J\left(\vartheta^{\mathrm{k}+1}\right)+\beta \mathrm{S}^{\mathrm{k}}, \quad \beta=\left\|\nabla J\left(\vartheta^{\mathrm{k}+1}\right)\right\|^{2} /\left\|\nabla J\left(\vartheta^{\mathrm{k}}\right)\right\|^{2},
\end{gathered}
$$

where the index $\mathrm{k}$ denotes the iteration number; $\vartheta^{0} \in R^{M \cdot m^{N}}$ is some admissible initial approximation of the optimized vector; $\nabla J\left(\vartheta^{k}\right)$ the gradient of the objective function of the problem; $\alpha_{\mathrm{k}}$ the step size taken to the minimum of the objective function in the direction of the vector $\mathrm{S}^{\mathrm{k}} ; \mathrm{P}_{(V)}($.$) the projection operator onto the admissible set$ $V$. If the domain of admissible controls $V$ has a complex boundary and the projection operator onto it has no constructive character, then to solve the posed problem, one can use methods of sequential unconstrained optimization (for example, methods of internal and external penalty functions) with the use of effective methods of unconstrained optimization of the first order such as quasi-Newtonian methods (Bazaraa, M. S., Sherali, H. D., \& Shetty, C. M., 2013). To construct iterative methods based on the above optimization methods, it is essential to have exact formulas for the components of the objective functional gradients in the space of the optimized parameters of the zonal controls. To this purpose, we derive formulas for the gradient of the objective function in the space of optimizable parameters. The derivation of these formulas is based on the technique for calculating the increment of the objective functional obtained by incrementing the optimized parameters (Panteleev A.V. and Letova T.A., 2015; Itkis, U., 1976; Aida-Zade, K. R., \& Kuliev, S. Z., 2008; Stewart, D. E., \& Anitescu, M., 2010). The complete derivation of these formulas is provided in the Appendix.

Let us denote by $\prod_{i_{1}, i_{2}, \ldots, i_{N}}(\vartheta) \subseteq[0, T]$ the time interval during which the vector $\tilde{u}(t)$ belongs to the $\left(i_{1}, i_{2}, \ldots, i_{N}\right)$ th phase parallelepiped under the chosen values of controls $\vartheta(t)$ and functions $g_{0}(x), g_{1}(t)$, and $g_{2}(t)$. Consider the following boundary-value problem adjoint to (1)-(4):

$$
\begin{aligned}
& \psi_{t}=-a^{2} \psi_{x x},(x, t) \in(0,1) \times[0, T), \\
& \psi(x, T)=-2\left[u\left(x, T ; \vartheta, g_{0}, g_{1}, g_{2}\right)-\bar{u}(x)\right], x \in[0,1], \\
& \psi(0, t)=\psi(1, t)=0, t \in[0, T) .
\end{aligned}
$$

Here $\psi(x, t)=\psi\left(x, t ; \vartheta, g_{0}, g_{1}, g_{2}\right)$ is the adjoint variable - the solution of the problem (11)-(13) under some specific values of the controls $\vartheta$ and initial-boundary functions $g_{0}(x), g_{1}(t)$, and $g_{2}(t)$, to which corresponds the solution $u\left(x, T ; \vartheta, g_{0}, g_{1}, g_{2}\right)$ of the initial- and boundary-value problem (1)-(4). The following theorem holds.

Theorem. The components of the gradient of the functional in the problem (1)-(4), in the space of piecewise constant controls (8) and (9), for an arbitrary control $\vartheta \in V$ 
are determined by the formula:

$$
\begin{aligned}
& \frac{\partial J(\vartheta)}{\partial \vartheta_{i_{1}, i_{2}, \ldots, i_{N}}^{j}} \\
& =\iint_{G_{0}} \int_{G_{1}} \int_{G_{2}}-\psi\left(\bar{x}_{i_{1}, i_{2}, \ldots, i_{N}}, t ; \vartheta, g_{0}, g_{1}, g_{2}\right) \mathrm{d} t \mathrm{~d} \Phi_{2}\left(g_{2}\right) \mathrm{d} \Phi_{1}\left(g_{1}\right) \mathrm{d} \Phi_{0}\left(g_{0}\right), \\
& i_{s} \in\{1,2, \ldots, m\}, s=1,2, \ldots, N, j=1,2, \ldots, M,
\end{aligned}
$$

where $\psi\left(x, t ; \vartheta, g_{0}, g_{1}, g_{2}\right)$ is the solution of the adjoint problem (11)-(13), corresponding to the current zonal control.

Using the formulas for the objective functional gradient (14), we can propose the following iterative algorithm for determining piecewise constant synthesizing controls based on first-order optimization methods.

Step 1. For the current admissible value of the vector $\vartheta(t)$ and all initial and boundary conditions $g_{0} \in G_{0}, g_{1} \in G_{1}$, and $g_{2} \in G_{2}$, we solve the direct initial-andboundary value problem concerning (1) by some numerical method and the trajectory $u\left(x, t ; \vartheta, g_{0}, g_{1}, g_{2}\right)$ is identified.

Step 2. We find the solution to the adjoint initial-and-boundary value problem (11)(13) corresponding to the direct problem's solution, and the trajectory $\psi\left(x, t ; \vartheta, g_{0}, g_{1}, g_{2}\right)$ is identified.

Step 3. We calculate the components of the objective functional gradient are calculated by formulas (14) using any quadrature formula.

Step 4. The new approximation of the control vector is calculated using first-order finite-dimensional optimization numerical procedures using the iterative gradient projection method (10).

If the optimality condition is not met or the iterative process ends (for example, when $\left|\alpha_{k}\right|<\varepsilon_{1}$ or $\left|J\left(\vartheta^{\mathrm{k}+1}\right)-J\left(\vartheta^{\mathrm{k}}\right)\right|<\varepsilon_{2}$, where $\varepsilon_{1}$ and $\varepsilon_{2}$ are given positive values), steps 1 4 are repeated.

The control system's quality with the use of zonal control actions described above is significantly affected by choice of both the number and the zones' structure (8) themselves. An increase in the number of zones due to their refinement can only decrease the objective function value. On the other hand, an increase in the number of zones leads to the fact that control actions can change their values more often in time, and, therefore, on the one hand, the robustness of the control system deteriorates; on the other hand, this leads to rapid wear and failure of the actuating mechanisms. Conversely, an increase in the size of the zones, i.e., a decrease in their number, on the one hand, worsens the controllability of the object, and with a small number of them, the object may become completely uncontrollable. On the other hand, this increases the objective functional value, i.e., the quality of control deteriorates. Taking these issues into account, the following approach is recommended, in which at first an initial value of $m$ is arbitrarily selected and some zones (8) are assigned. Having solved the above control synthesis problem, we can analyze the computed optimal 
zonal values of the controls for all neighboring zones. If the optimized parameters in any two adjacent zones differ by a sufficiently small amount, then these adjacent zones can be combined into one, thus reducing the number $m$, the number of switchings of the control. If the optimized parameters in two adjacent zones differ significantly, then, on the contrary, each of these adjacent zones should be divided, for example, into two zones, i.e., increase the number $m$, and again solve the considered control synthesis problem. An increase in the number of zones should be carried out until the objective functional value ceases to change (decrease) significantly.

Remark 1. The frequency of observation times $\tau_{i} \in[0, T](i=0,1, \ldots, q)$ should be such that while the object's state belongs to any zone, at least one observation is made. If this condition is not met, the zones through which the system's trajectory did not pass under all possible initial and boundary conditions, as well as the zones through which no state measurements were carried out, will not be assigned the values of the zonal control parameters.

Remark 2. The main issue with the proposed approach to feedback is the highdimensionality of the optimizable control vector. The optimizable control vector's dimension represents a power function with respect to the number $m$ of temperature intervals within the range $\left[u_{\min }, u_{\mathrm{max}}\right]$ of all possible temperature values of the object, and an exponential function with respect to the number of thermal sensors installed along the length of the rod. Besides that, the number of thermal sources also affects the optimizable control vector (as a multiplication factor of the term $\mathrm{m}^{N}$ ). It is known that one of the basic problems of numerical optimization techniques (of any order) is the computation of optimal solutions of high-dimensional objective functions. This is because the optimization of high-dimensional objective functions is computationally expensive and cost involved, especially when seeking the global optimal solution. Many parameters characterize these kinds of problems, and many iterations and arithmetic operations are usually needed for evaluations of these objective functions. In order to speed up the evaluation of the objective functional in the posed optimal feedback control problem, under the given value of the control vector, we can make use of the inherent concurrency present in the form of the objective functional. Namely, because the evaluation of the objective functional involves the computation of definite integral (6), knowing that the elements of the sets $G_{0}, G_{1}$, and $G_{2}$ are independent, we can efficiently parallelize its computation by assigning to each thread (or process) a specific triplet of functions $g_{0}, g_{1}$, and $g_{2}$, and computing the innermost definite integral in (6) with sufficiently high accuracy. The innermost integration can also be parallelized if we preliminarily slice the interval $[0,1]$ into several non-overlapping subintervals and computing the definite integral over all these subintervals concurrently (Deng, Y., 2012). The same concurrency pattern also applies to evaluating the gradient of the objective functional by the formula (14). Note that the solution to both the direct and adjoint initial-and-boundary value problems with respect to the parabolic type differential equation can be easily parallelized, too, if we employ an explicit finite 
difference scheme to their solution (Deng, Y., 2012; Dongarra, J., Foster, I., Fox, G., Gropp, W., Kennedy, K., Torczon, L., \& White, A., 2003). Even if employ implicit finite difference schemes to their solution, we will still be able to parallelize computations using efficient iterative methods of solution to linear systems with banded coefficient matrices.

\section{Conclusion}

In this work, we have obtained formulas for the gradient of the objective function in synthesizing optimal zonal control of an object described by a system of differential equations with partial derivatives given inaccurate information on the values of the initial and boundary conditions of the object. The formulas obtained make it possible to apply first-order finite-dimensional optimization methods for the numerical solution of the problems under consideration. It is known that the technical implementation of piecewise-constant synthesizing functions with sufficiently high accuracy is relatively simple. Therefore, the proposed approach to solving optimal control synthesis problems can find wide application in automated systems and automatic control of systems in the presence of inaccurate information on the object's state. The application objects can be many controlled mechanical systems, technological processes described by systems of nonlinear differential equations. Note that the proposed approach can easily be extended to two- and three-dimensional heat conduction processes.

\section{Appendix}

Without loss of generality of the reasoning given below, to avoid the cumbersomeness of the resulting formulas, we consider the case when there is only one control function, i.e., $M=1$ (we will henceforth omit the superscript of the function $\vartheta^{1}(t)$, i.e., denote it by $\left.\vartheta(t)\right)$. In further calculations, the following remark is important. The initial and boundary conditions (2)-(4), i.e., the elements of the sets $G_{0}(x), G_{1}(t)$, and $G_{2}(t)$ are independent. Then the gradient of the functional satisfies the formula

$$
\nabla J(\vartheta)=\int_{G_{0}} \int_{G_{1}} \int_{G_{2}} \nabla I\left(x, T ; \vartheta, g_{0}, g_{1}, g_{2}\right) \mathrm{d} \Phi_{2}\left(g_{2}\right) \mathrm{d} \Phi_{1}\left(g_{1}\right) \mathrm{d} \Phi_{0}\left(g_{0}\right)
$$

Therefore, starting now, to obtain formulas for $\nabla J(\vartheta)$, we obtain formulas for the gradient of $I($.$) with respect to individual terms g_{0}(x), g_{1}(t)$, and $g_{2}(t)$, (i.e., assuming that each of the sets $G_{0}(x), G_{1}(t)$, and $G_{2}(t)$ consists of a single term). For this purpose, we obtain the formula for the increment of the functional (7), obtained by incrementing the parameter value $\vartheta=\left(\vartheta_{i_{1}, i_{2}, \ldots, i_{N}}\right)=\left(\vartheta_{1}, \vartheta_{2}, \ldots, \vartheta_{p}\right)^{*}, i_{s} \in\{1,2, \ldots, m\}, s=1,2, \ldots, N$. Suppose that one of the optimizable parameters of the vector $\vartheta$ is incremented, for example, $\vartheta_{k}$, i.e.

$$
\bar{\vartheta}=\vartheta+\Delta \vartheta, \quad \Delta \vartheta=\left(0,0, \ldots, 0, \Delta \vartheta_{k}, 0, \ldots, 0\right)^{*},
$$


where $\bar{\vartheta}$ denotes the perturbed control action.

To express the increment of the functional (7) in terms of the increment of the control action, $\Delta \vartheta$, we introduce the Lagrangian for the considered problem:

$$
\begin{aligned}
L(u, \psi ; \vartheta)=\int_{0}^{1}[u(x, T)-\hat{u}(x)]^{2} \mathrm{~d} x \\
\quad+\int_{0}^{T} \int_{0}^{1}\left[u_{t}-a^{2} u_{x x}-\vartheta(t) \delta(x-\bar{x})\right] \psi(x, t) \mathrm{d} x \mathrm{~d} t
\end{aligned}
$$

where $u(x, T)=u\left(x, T ; \vartheta, g_{0}, g_{1}, g_{2}\right)$ denotes the solution of PDE

$$
u_{t}=a^{2} u_{x x}+\vartheta(t) \delta(x-\bar{x})
$$

at the final moment of time $t=T$, satisfying the initial and boundary conditions

$$
\begin{aligned}
& u(x, 0)=g_{0}(x), x \in[0,1], \\
& u(0, t)=g_{1}(t), t \in[0, T], \\
& u(1, t)=g_{2}(t), t \in[0, T],
\end{aligned}
$$

and $\psi=\psi(x, t)$ is yet an arbitrary smooth function.

Assume that at the moment of time $\tau_{k}$, the measurements taken at the thermal sensors indicate that the object's state belongs to the $k$ th zone, while at any last moments of time, $\tau_{0}, \tau_{1}, \ldots, \tau_{k-1}$, the object's state does not belong to the $k$ th zone. Then we can rewrite (15) as follows:

$$
\begin{aligned}
L(u, \psi ; \theta) & =\int_{0}^{1}[u(x, T)-\hat{u}(x)]^{2} \mathrm{~d} x \\
& \quad+\int_{0}^{\tau_{k}} \int_{0}^{1}\left[u_{t}-a^{2} u_{x x}-\vartheta(t) \delta(x-\bar{x})\right] \psi \mathrm{d} x \mathrm{~d} t+ \\
+ & \int_{\tau_{k}}^{\tau_{k+l}} \int_{0}^{1}\left[u_{t}-a^{2} u_{x x}-\vartheta_{k} \delta(x-\bar{x})\right] \psi \mathrm{d} x \mathrm{~d} t+ \\
+ & \int_{\tau_{k+l}}^{T} \int_{0}^{1}\left[u_{t}-a^{2} u_{x x}-\vartheta(t) \delta(x-\bar{x})\right] \psi \mathrm{d} x \mathrm{~d} t
\end{aligned}
$$

Here $\tau_{k+l}$ denotes the last moment at which measurements taken at the thermal sensors indicate that the object's state belongs to the $k$ th zone.

Let's denote by $\bar{u}(x, t)=u(x, t)+\Delta u(x, t)$ the solution of the PDE (16) corresponding to the perturbed control $\bar{\vartheta}$ and satisfying the same initial and boundary conditions (17)-(19). We assume that the increment $\Delta \vartheta$ of the control is so small (which ultimately is due to the definition of the derivative) that the measurements were taken at the thermal sensors during the time interval $\left[\tau_{k}, \tau_{k+l}\right]$ still indicates that the object's state belongs to the $k$ th zone. Under these assumptions, the Lagrangian (20) for the perturbed solution $\bar{u}(x, t)$ takes on the following form: 


$$
\begin{aligned}
& L(\bar{u}, \psi ; \bar{\vartheta})=\int_{0}^{1}[\bar{u}(x, T)-\hat{u}(x)]^{2} \mathrm{~d} x \\
& \quad+\int_{0}^{\tau_{k}} \int_{0}^{1}\left[\bar{u}_{t}-a^{2} \bar{u}_{x x}-\vartheta(t) \delta(x-\bar{x})\right] \psi \mathrm{d} x \mathrm{~d} t+ \\
& +\int_{\tau_{k}}^{\tau_{k+l}} \int_{0}^{1}\left[\bar{u}_{t}-a^{2} \bar{u}_{x x}-\left(\vartheta_{k}+\Delta \vartheta_{k}\right) \delta(x-\bar{x})\right] \psi \mathrm{d} x \mathrm{~d} t+ \\
& \quad+\int_{\tau_{k+l}}^{T} \int_{0}^{1}\left[\bar{u}_{t}-a^{2} \bar{u}_{x x}-\vartheta(t) \delta(x-\bar{x})\right] \psi \mathrm{d} x \mathrm{~d} t
\end{aligned}
$$

Subtracting (21) from (20) produces the increment of the Lagrangian:

$$
\begin{aligned}
& \Delta L=L(\bar{u}, \psi ; \bar{\vartheta})-L(u, \psi ; \vartheta) \\
& \quad=\int_{0}^{1}[u(x, T)+\Delta u(x, T)-\hat{u}(x)]^{2} \mathrm{~d} x-\int_{0}^{1}[u(x, T)-\hat{u}(x)]^{2} \mathrm{~d} x+ \\
& \quad+\int_{0}^{\tau_{k}} \int_{0}^{1}\left[u_{t}+\Delta u_{t}-a^{2} u_{x x}-a^{2} \Delta u_{x x}-\vartheta(t) \delta(x-\bar{x})\right] \psi \mathrm{d} x \mathrm{~d} t+ \\
& +\int_{\tau_{k}}^{\tau_{k+l}} \int_{0}^{1}\left[u_{t}+\Delta u_{t}-a^{2} u_{x x}-a^{2} \Delta u_{x x}-\left(\vartheta_{k}+\Delta \vartheta_{k}\right) \delta(x-\bar{x})\right] \psi \mathrm{d} x \mathrm{~d} t+ \\
& +\int_{\tau_{k+l}}^{T} \int_{0}^{1}\left[u_{t}+\Delta u_{t}-a^{2} u_{x x}-a^{2} \Delta u_{x x}-\vartheta(t) \delta(x-\bar{x})\right] \psi \mathrm{d} x \mathrm{~d} t- \\
& \quad-\int_{0}^{\tau_{k}} \int_{0}^{1}\left[u_{t}-a^{2} u_{x x}-\vartheta(t) \delta(x-\bar{x})\right] \psi \mathrm{d} x \mathrm{~d} t \\
& -\int_{\tau_{k+l}}^{\tau_{k+l}} \int_{0}^{1}\left[u_{t}-a^{2} u_{x x}-\vartheta(t) \delta(x-\bar{x})\right] \psi \mathrm{d} x \mathrm{~d} t
\end{aligned}
$$

We denote by $\Delta_{1} L$ the sum of the first two terms and by $\Delta_{2} L$ the sum of all the other terms in the last expression. First, we modify $\Delta_{1} L$ :

$$
\begin{gathered}
\Delta_{1} L=\int_{0}^{1}[u(x, T)-\tilde{u}(x)+\Delta u(x, T)]^{2} \mathrm{~d} x-\int_{0}^{1}[u(x, T)-\tilde{u}(x)]^{2} \mathrm{~d} x= \\
=\int_{0}^{1}[u(x, T)-\tilde{u}(x)]^{2} \mathrm{~d} x+\int_{0}^{1} 2[u(x, T)-\tilde{u}(x)] \Delta u(x, T) \mathrm{d} x+\int_{0}^{1}[\Delta u(x, T)]^{2} \mathrm{~d} x- \\
-\int_{0}^{1}[u(x, T)-\tilde{u}(x)]^{2} \mathrm{~d} x
\end{gathered}
$$

After canceling out the similar terms, we obtain:

$$
\Delta_{1} L=\int_{0}^{1} 2[u(x, T)-\tilde{u}(x)] \Delta u(x, T) \mathrm{d} x+\int_{0}^{1}[\Delta u(x, T)]^{2} \mathrm{~d} x .
$$


Then, we modify $\Delta_{2} L$ :

$$
\begin{gathered}
\Delta_{2} L=\int_{0}^{\tau_{k}} \int_{0}^{1}\left[u_{t}-a^{2} u_{x x}-\vartheta(t) \delta(x-\bar{x})\right] \psi \mathrm{d} x \mathrm{~d} t+\int_{0}^{\tau_{k}} \int_{0}^{1}\left[\Delta u_{t}-a^{2} \Delta u_{x x}\right] \psi \mathrm{d} x \mathrm{~d} t+ \\
\quad+\int_{\tau_{k}}^{\tau_{k+l}} \int_{0}^{1}\left[u_{t}-a^{2} u_{x x}-\vartheta_{k} \delta(x-\bar{x})\right] \psi \mathrm{d} x \mathrm{~d} t+ \\
\quad+\int_{\tau_{k}}^{\tau_{k+l}} \int_{0}^{1}\left[\Delta u_{t}-a^{2} \Delta u_{x x}-\Delta \vartheta_{k} \delta(x-\bar{x})\right] \psi \mathrm{d} x \mathrm{~d} t+ \\
+\int_{\tau_{k+l}}^{T} \int_{0}^{1}\left[u_{t}-a^{2} u_{x x}-\vartheta(t) \delta(x-\bar{x})\right] \psi \mathrm{d} x \mathrm{~d} t+\int_{\tau_{k+l}}^{T} \int_{0}^{1}\left[\Delta u_{t}-a^{2} \Delta u_{x x}\right] \psi \mathrm{d} x \mathrm{~d} t- \\
-\int_{0}^{\tau_{k}} \int_{0}^{1}\left[u_{t}-a^{2} u_{x x}-\vartheta(t) \delta(x-\bar{x})\right] \psi \mathrm{d} x \mathrm{~d} t \\
-\int_{\tau_{k}}^{\tau_{k+l}} \int_{0}^{1}\left[u_{t}-a^{2} u_{x x}-\vartheta_{k} \delta(x-\bar{x})\right] \psi \mathrm{d} x \mathrm{~d} t- \\
-\int_{\tau_{k+l}}^{T} \int_{0}^{1}\left[u_{t}-a^{2} u_{x x}-\vartheta(t) \delta(x-\bar{x})\right] \psi \mathrm{d} x \mathrm{~d} t
\end{gathered}
$$

After cancelling out the similar terms, we obtain:

$$
\begin{gathered}
\Delta_{2} L=\int_{0}^{\tau_{k}} \int_{0}^{1}\left[\Delta u_{t}-a^{2} \Delta u_{x x}\right] \psi \mathrm{d} x \mathrm{~d} t+\int_{\tau_{k}}^{\tau_{k+l}} \int_{0}^{1}\left[\Delta u_{t}-a^{2} \Delta u_{x x}\right] \psi \mathrm{d} x \mathrm{~d} t+ \\
+\int_{\tau_{k+l}}^{T} \int_{0}^{1}\left[\Delta u_{t}-a^{2} \Delta u_{x x}\right] \psi \mathrm{d} x \mathrm{~d} t+\int_{\tau_{k}}^{\tau_{k+l}} \int_{0}^{1}\left[-\Delta \vartheta_{k} \delta(x-\bar{x})\right] \psi \mathrm{d} x \mathrm{~d} t
\end{gathered}
$$

By combining the first three terms of the last expression, we obtain:

$$
\Delta_{2} L=\int_{0}^{T} \int_{0}^{1}\left[\Delta u_{t}-a^{2} \Delta u_{x x}\right] \psi \mathrm{d} x \mathrm{~d} t+\int_{\tau_{k}}^{\tau_{k+l}} \int_{0}^{1}\left[-\Delta \vartheta_{k} \delta(x-\bar{x})\right] \psi \mathrm{d} x \mathrm{~d} t
$$

Taking $\Delta_{1} L$ and $\Delta_{2} L$ into account in $\Delta L$, we obtain:

$$
\begin{gathered}
\Delta L=\int_{0}^{1} 2[u(x, T)-\tilde{u}(x)] \Delta u(x, T) \mathrm{d} x+\int_{0}^{T} \int_{0}^{1}\left[\Delta u_{t}-a^{2} \Delta u_{x x}\right] \psi \mathrm{d} x \mathrm{~d} t+ \\
+\int_{0}^{1}[\Delta u(x, T)]^{2} \mathrm{~d} x+\int_{\tau_{k}}^{\tau_{k+l}} \int_{0}^{1}\left[-\Delta \vartheta_{k} \delta(x-\bar{x})\right] \psi \mathrm{d} x \mathrm{~d} t
\end{gathered}
$$

We have managed to extract the linear (with respect to $\Delta \vartheta_{k}$ ) part of the Lagrangian increment. In order to obtain the expression for the derivative of $L($.$) with respect to$ $\Delta \vartheta_{k}$, we have to eliminate the first two terms of (22). In order to do that, we modify the second term of (22) by breaking it down into two terms:

$$
\int_{0}^{T} \int_{0}^{1}\left[\Delta u_{t}-a^{2} \Delta u_{x x}\right] \psi \mathrm{d} x \mathrm{~d} t=\int_{0}^{T} \int_{0}^{1} \Delta u_{t} \psi \mathrm{d} x \mathrm{~d} t-\int_{0}^{T} \int_{0}^{1} a^{2} \Delta u_{x x} \psi \mathrm{d} x \mathrm{~d} t
$$

We apply the integration by parts technique to both the integrals. For the first of these integrals, we have: 


$$
\begin{gathered}
\int_{0}^{T} \int_{0}^{1} \Delta u_{t} \psi \mathrm{d} x \mathrm{~d} t=\int_{0}^{1}\left\{\int_{0}^{T} \Delta u_{t} \psi \mathrm{d} t\right\} \mathrm{d} x=\int_{0}^{1}\left\{\int_{0}^{T} \psi \mathrm{d}_{\mathrm{t}} \Delta u\right\} \mathrm{d} x= \\
=\int_{0}^{1}\left\{\left.\psi \Delta u\right|_{0} ^{T}-\int_{0}^{T} \Delta u \mathrm{~d}_{\mathrm{t}} \psi\right\} \mathrm{d} x \\
=\int_{0}^{1}\left\{\psi(x, T) \Delta u(x, T)-\psi(x, 0) \Delta u(x, 0)-\int_{0}^{T} \Delta u \psi_{t} \mathrm{dt}\right\} \mathrm{d} x
\end{gathered}
$$

According to initial condition (17), we have:

$\Delta u(x, 0)=\bar{u}(x, 0)-u(x, 0)=g_{0}(x)-g_{0}(x)=0$ for all $x \in[0,1]$.

Therefore

$$
\int_{0}^{T} \int_{0}^{1} \Delta u_{t} \psi \mathrm{d} x \mathrm{~d} t=\int_{0}^{1} \psi(x, T) \Delta u(x, T) \mathrm{d} x-\int_{0}^{T} \int_{0}^{1} \Delta u \psi_{t} \mathrm{~d} x \mathrm{~d} t
$$

For the second integral, we have:

$$
\begin{gathered}
-\int_{0}^{T} \int_{0}^{1} a^{2} \Delta u_{x x} \psi \mathrm{d} x \mathrm{~d} t=-a^{2} \int_{0}^{T}\left\{\int_{0}^{1} \psi \mathrm{d}_{\mathrm{x}} \Delta u_{x}\right\} \mathrm{d} t= \\
=-a^{2} \int_{0}^{T}\left\{\left.\psi \Delta u_{x}\right|_{0} ^{1}-\int_{0}^{1} \Delta u_{x} \mathrm{~d}_{x} \psi\right\} \mathrm{d} t= \\
=a^{2} \int_{0}^{T}\left\{\psi(0, t) \Delta u_{x}(0, t)-\psi(1, t) \Delta u_{x}(1, t)+\int_{0}^{1} \Delta u_{x} \psi_{x} \mathrm{~d} x\right\} \mathrm{d} t
\end{gathered}
$$

Due to the arbitrariness of the function $\psi(x, t)$, we require that

$$
\psi(0, t)=\psi(1, t)=0 \text { for all } t \in[0, T]
$$

Then the last expression reduces to:

$$
-\int_{0}^{T} \int_{0}^{1} a^{2} \Delta u_{x x} \psi \mathrm{d} x \mathrm{~d} t=a^{2} \int_{0}^{T}\left\{\int_{0}^{1} \Delta u_{x} \psi_{x} \mathrm{~d} x\right\} \mathrm{d} t,
$$

which we can further modify by applying the integration by parts technique again. We thus obtain:

$$
\begin{gathered}
a^{2} \int_{0}^{T}\left\{\int_{0}^{1} \Delta u_{x} \psi_{x} \mathrm{~d} x\right\} \mathrm{d} t=a^{2} \int_{0}^{T}\left\{\int_{0}^{1} \psi_{x} \mathrm{~d}_{\mathrm{x}} \Delta u\right\} \mathrm{d} t= \\
=a^{2} \int_{0}^{T}\left\{\left.\psi_{x} \Delta u\right|_{0} ^{1}-\int_{0}^{1} \Delta u \mathrm{~d}_{\mathrm{x}} \psi_{x}\right\} \mathrm{d} t= \\
=a^{2} \int_{0}^{T}\left\{\psi_{x}(1, t) \Delta u(1, t)-\psi_{x}(0, t) \Delta u(0, t)-\int_{0}^{1} \Delta u \psi_{x x} \mathrm{~d} x\right\} \mathrm{d} t
\end{gathered}
$$

According to boundary conditions (18) and (19), we have:

$\Delta u(0, t)=\bar{u}(0, t)-u(0, t)=g_{1}(t)-g_{1}(t)$ for all $t \in[0, T]$,

$\Delta u(1, t)=\bar{u}(1, t)-u(1, t)=g_{2}(t)-g_{2}(t)$ for all $t \in[0, T]$.

Therefore:

$$
-\int_{0}^{T} \int_{0}^{1} a^{2} \Delta u_{x x} \psi \mathrm{d} x \mathrm{~d} t=-\int_{0}^{T} \int_{0}^{1} a^{2} \psi_{x x} \Delta u \mathrm{~d} x \mathrm{~d} t
$$


Taking (23) and (25) into account in (22) produces:

$$
\begin{gathered}
\Delta L=\int_{0}^{1} 2[u(x, T)-\tilde{u}(x)] \Delta u(x, T) \mathrm{d} x+\int_{0}^{1}[\Delta u(x, T)]^{2} \mathrm{~d} x+ \\
+\int_{0}^{1} \psi(x, T) \Delta u(x, T) \mathrm{d} x-\int_{0}^{T} \int_{0}^{1} \psi_{t} \Delta u \mathrm{~d} x \mathrm{~d} t- \\
-\int_{0}^{T} \int_{0}^{1} a^{2} \psi_{x x} \Delta u \mathrm{~d} x \mathrm{~d} t+\int_{\tau_{k}}^{\tau_{k+l}} \int_{0}^{1} \psi\left[-\Delta \theta_{k} \delta(x-\bar{x})\right] \mathrm{d} x \mathrm{~d} t
\end{gathered}
$$

By combining the 1st with the 3rd terms, and the 4th with the 5th terms, we obtain:

$$
\begin{aligned}
\Delta L & =\int_{0}^{1}\{\psi(x, T)+2[u(x, T)-\tilde{u}(x)]\} \Delta u(x, T) \mathrm{d} x+\int_{0}^{1}[\Delta u(x, T)]^{2} \mathrm{~d} x- \\
& -\int_{0}^{T} \int_{0}^{1}\left\{\psi_{t}+a^{2} \psi_{x x}\right\} \Delta u \mathrm{~d} x \mathrm{~d} t+\int_{\tau_{k}}^{\tau_{k+l}} \int_{0}^{1} \psi\left[-\Delta \theta_{k} \delta(x-\bar{x})\right] \mathrm{d} x \mathrm{~d} t
\end{aligned}
$$

Assume that:

$$
\begin{array}{r}
\psi(x, T)=-2[u(x, T)-\tilde{u}(x)] \text { for all } x \in[0,1], \\
\psi_{t}=-a^{2} \psi_{x x} \text { for }(x, t) \in \Omega .
\end{array}
$$

Then the increment of the Lagrangian takes on the following form:

$$
\Delta L=\int_{\tau_{k}}^{\tau_{k+l}} \int_{0}^{1} \psi\left[-\Delta \vartheta_{k} \delta(x-\bar{x})\right] \mathrm{d} x \mathrm{~d} t+\int_{0}^{1}[\Delta u(x, T)]^{2} \mathrm{~d} x
$$

Because $\Delta \vartheta_{k}$ is constant, we can take it out of the integral sign, thus obtaining:

$$
\Delta L=\int_{\tau_{k}}^{\tau_{k+l}} \int_{0}^{1}-\psi \delta(x-\bar{x}) \mathrm{d} x \mathrm{~d} t \Delta \vartheta_{k}+\int_{0}^{1}[\Delta u(x, T)]^{2} \mathrm{~d} x .
$$

Dropping the second term of the last expression and taking into account the definition of Dirac's delta function, we obtain the expression for the derivative of $L(u, \psi ; \theta)$ with respect to $\theta_{k}$ :

$$
\frac{\partial L(u, \psi ; \theta)}{\partial \theta_{k}}=\int_{\tau_{k}}^{\tau_{k+l}}-\psi(\bar{x}, t) \mathrm{d} t
$$

Remark 3. In general, the object's state at the observed points can belong to the same zone in disparate time intervals $\left[\tau_{i}, \tau_{i+1}\right), i \in\{0,1,2, \ldots, q-1\}$, i.e. the trajectory of the system may lie in the same zone again and again in different points of time $\tau_{i}$. In this case, we have to modify the formula (28) by calculating the definite integral over the combined time interval

$$
\prod_{i_{1}, i_{2}, \ldots, i_{N}}(\vartheta)=\bigcup_{\widetilde{u}\left(\tau_{i}\right) \in P_{i_{1}, i_{2}, \ldots, i_{N}}}\left[\tau_{i}, \tau_{i+1}\right)
$$

during which the vector $\tilde{u}(t)$ belongs to the $\left(i_{1}, i_{2}, \ldots, i_{N}\right)$ th phase parallelepiped under the chosen values of controls $\vartheta(t)$ and functions $g_{0}(x), g_{1}(t)$, and $g_{2}(t)$. The formula (28) should be modified accordingly: 


$$
\frac{\partial L(u, \psi ; \theta)}{\partial \theta_{k}}=\int_{\prod_{i_{1}, i_{2}, \ldots, i_{N}}(\vartheta)}-\psi(\bar{x}, t) \mathrm{d} t
$$

Generalizing this formula to all possible states of the initial and boundary conditions, i.e., covering the entire sets $G_{0}(),. G_{1}($.$) , and G_{2}($.$) , we arrive at formula (14).$

\section{References}

Aida-Zade, K. R., Kuliev, S. Z. (2008). A class of inverse problems for discontinuous systems. Cybernetics and Systems Analysis, 44(6), 915-924.

Aida-Zade, K. R., Kuliev, S. Z. (2011). Numerical solution of nonlinear inverse coefficient problems for ordinary differential equations. Computational Mathematics and Mathematical Physics, 51(5), 803-815.

Aida-Zade, K. R., Kuliev, S. Z. (2012). On numerical solution of one class of inverse problems for discontinuous dynamic systems. Automation and Remote Control, 73(5), 786-796.

Arthur, E., Bryson, Yu-Chi Ho. (1975) Applied optimal control: Optimization, estimation and control. CRC Press; $1^{\text {st }}$ edt., 1975.

Bazaraa, M. S., Sherali, H. D., \& Shetty, C. M. (2013). Nonlinear programming: theory and algorithms. John Wiley \& Sons.

Butkovsky, A.G. (1975) Methods for controlling systems with distributed parameters. Moscow: Nauka. (in Russian)

Deng, Y. (2012). Applied parallel computing. World Scientific.

Dongarra, J., Foster, I., Fox, G., Gropp, W., Kennedy, K., Torczon, L., \& White, A. (2003). Sourcebook of parallel computing (Vol. 3003). San Francisco eCA CA: Morgan Kaufmann Publishers.

Egorov, A.I. (2004) Foundations of control theory. Moscow: Fizmatlit. (in Russian)

Fursikov, A.V. (1999) Optimal control of distributed systems: Theory and applications. American Mathematical Society.

Guliyev, S. Z. (2013). Synthesis of control in nonlinear systems with different types of feedback and strategies of control. Journal of Automation and Information Sciences, 45(7), 74-86.

Guliyev, S. Z. (2018). Numerical solution of a zonal feedback control problem for the heating process. IFAC-PapersOnLine, 51(30), 251-256.

Guliyev, S.Z., Aida-zade, K.R. (2005) Optimization of location and operation modes of oilfield wells. Computational Technologies SB RAS, 10 (4), 52-62. (in Russian)

Guliyev, S.Z., Aida-zade, K.R. (2016) Hydraulic resistance coefficient identification in pipelines. Automation and Remote Control, 77 (7), 1225-1239.

Itkis, U. (1976). Control systems of variable structure. Halsted Press.

Kuliev, S. Z. (2011). Synthesis of zonal controls of nonlinear systems under discrete observations. Automatic Control and Computer Sciences, 45(6), 338-345.

Lions, J.L. (1971) Optimal control of systems governed by partial differential 
equations. Springer-Verlag (Berlin).

Lurie, K.A. (1993) Applied optimal control theory of distributed systems. Springer US.

Moiseev, N.N. (1971) Numerical methods in the theory of optimal systems. Moscow: Nauka. (in Russian)

Nocedal, J., Wright, S. (2006). Numerical optimization. Springer Science \& Business Media.

Panteleev, A.V. Letova, T.A. (2015) Optimization methods in examples and problems. Saint-Petersburg: Lan Publishing. (in Russian)

Rapoport, E.Ya. (2009) Optimal control of systems with distributed parameters. Moscow: Higher school. (in Russian)

Sirazetdinov, T.K. (1977) Optimization of systems with distributed parameters. Moscow: Nauka. (in Russian)

Stewart, D. E., Anitescu, M. (2010). Optimal control of systems with discontinuous differential equations. Numerische Mathematik, 114(4), 653-695.

Vasiliev, F.P. (2002) Optimization methods. Moscow: Factorial Press, 2002. (in Russian)

Submitted: 02.05.2020

Accepted: 25.11.2020 